\title{
Análisis comparativo del plan de estudios de contaduría pública de la FACEM-UNICAN con las normas internacionales de formación emitidas por la IFAC-año 2021
}

\author{
Derlis Daniel Duarte Sánchez \\ derlisduarte@facem.edu.py \\ https://orcid.org/0000-0002-6717-2873 \\ Doctorando en Contabilidad y Auditoría. \\ Universidad Nacional de Canindeyú \\ Facultad de Ciencias Económicas y Empresariales \\ Salto del Guairá-Paraguay
}

\section{RESUMEN}

En la investigación se tiene como propósito verificar si el programa de estudios de Contaduría Pública de la Facultad de Ciencias Económicas y Empresariales de la Universidad Nacional de Canindeyú cumple con las condiciones necesarias para generar las competencias requeridas para el ejercicio profesional de sus egresados de acuerdo Consejo de Normas Internacionales de Formación en Contaduría específicamente de los contenidos de los programas profesionales de formación en contaduría. Se utilizó una metodología de enfoque cualitativo, de diseño no experimental, de nivel descriptivo, de corte transversal, la modalidad fue documental, la técnica de recolección de datos fue el análisis documental de los textos, el instrumento la ficha bibliográfica. Como resultado se comprueba que la carrera de Contaduría Pública cumple plenamente las recomendaciones del manual de los pronunciamientos internacionales, específicamente de los contenidos de los programas profesionales de formación en contaduría. Se recomienda a la comunidad académica (estudiantes, docentes, directivos y empleadores) seguir con la excelencia académica a través de un esfuerzo en conjunto actualizando los contenidos programáticos en consonancia a las demandas sociales.

Palabras clave: contaduría pública; ejercicio profesional; normas internacionales de contabilidad; calidad educativa. 


\title{
Comparative analysis of the FACEM-UNICAN public accounting curriculum with the international training standards issued by IFAC-year 2021
}

\begin{abstract}
The purpose of the investigation is to verify if the Public Accounting study program of the Faculty of Economic and Business Sciences of the National University of Canindeyú meets the necessary conditions to generate the skills required for the professional practice of its graduates according to the Council of International Accounting Education Standards specifically on the contents of professional accounting education programs. A qualitative approach methodology was used, with a non-experimental design, descriptive level, cross-sectional, the modality was documentary, the data collection technique was the documentary analysis of the texts, the instrument the bibliographic record. As a result, it is verified that the Public Accounting career fully complies with the recommendations of the manual of international pronouncements, specifically the contents of professional accounting training programs. The academic community (students, teachers, directors and employers) is recommended to continue with academic excellence through a joint effort updating the program content in line with social demands.
\end{abstract}

Keywords: public accounting; professional practice; international accounting standards; educational quality.

Artículo recibido: 02 enero 2022

Aceptado para publicación: 28 enero 2022 Correspondencia: derlisduarte@ facem.edu.py Conflictos de Interés: Ninguna que declarar

\section{INTRODUCCIÓN}

En entornos cada vez más globalizados, donde el conocimiento es cada vez más dinámico, las instituciones de educación superior no pueden estar aletargadas y estáticas, sino por 
el contrario, deben estar a la vanguardia en diversos aspectos, entre ellos, las mallas curriculares (Salvioni Durañona, 2020, p. 1).

Las principales preocupaciones sobre educación contable han girado alrededor de temáticas como modelos pedagógicos, currículo y preparación docente, lo cual ha causado una construcción del discurso pedagógico en América Latina, algunos países de Europa como España y Francia y ciertas posturas norteamericanas, pudiendo destacar que se comparten significativamente referencias y lineamientos para construcciones interdisciplinares y transdisciplinares (García Jiménez, 2014, p. 8).

La misión de la Federación Internacional de Contadores (International Federation of Accountants /IFAC) es servir al interés público, fortalecer la profesión contable en todo el mundo y contribuir al desarrollo de economías internacionales, estableciendo normas profesionales de alta calidad, fomentando su cumplimiento, favoreciendo su convergencia internacional, y manifestándose sobre aquellos temas de interés público para los que la experiencia de la profesión sea más relevante (Federación Internacional de Contadores \& Consejo de Normas Internacionales de Formación en Contaduría, 2008, p. 2).

El Consejo de Normas Internacionales de Formación en Contaduría (International Accounting Education Standards Board/IAESB), un Consejo emisor de Normas independiente, dentro de la Federación Internacional de Contadores, desarrolla normas y guías sobre la precalificación de formación, capacitación, y desarrollo profesional continuo para todos los miembros de la profesión contable. El Consejo de Supervisión del Interés Público (Public Interest Oversight Board/PIOB) para la profesión contable supervisa las actividades del IAESB(Federación Internacional de Contadores \& Consejo de Normas Internacionales de Formación en Contaduría, 2008, p. 2).

Por lo expuesto, la carrera de Contaduría Pública tiene como misión formar profesionales en las distintas áreas de la contabilidad, con elevados estándares de calidad, en consonancia con las demandas sociales. Producir conocimientos científicos, útiles a la sociedad. Conservar la cultura nacional y universal. Promover el desarrollo económico y social en su área de influencia y contribuir en la mejora de la calidad de vida de los habitantes del Departamento de Canindeyú, de su zona de influencia y del país (Facultad 
de Ciencias Económicas y Empresariales de la Universidad Nacional de Canindeyú, 2011).

El propósito del presente trabajo es comparar la malla curricular de carrera con la recomendación del manual de los pronunciamientos internacionales de formación emitida por la Federación Internacional de Contadores (IFAC) a través del Consejo de Normas Internacionales de Formación en Contaduría (International Accounting Education Standards Board/IAESB) si el programa de estudios de la carrera cumple con los requerimientos.

\section{MATERIALES Y MÉTODOS}

El enfoque de la investigación es cualitativo porque se utiliza la recolección y análisis de los datos para afinar las preguntas de investigación o revelar nuevas interrogantes en el proceso de interpretación, básicamente no se utilizan datos numéricos (Hernandez Sampieri et al., 2014, p. 7)

El diseño de investigación es no experimental porque no se realiza ningún tipo de experimento (Hernandez Sampieri \& Mendoza Torres, 2018, p. 174), además, el nivel de investigación es descriptivo porque se describe características importantes de la carrera de Contaduría Pública (Peña Marroquín, 2018, p. 4).

Por tanto, el tiempo de la investigación es de corte trasversal porque la recolección de datos se realiza en un momento dado (Müggenburg \& Pérez, 2018, p. 4). La modalidad es documental porque para la recolección de datos se realiza a través revisión documental (fichas bibliográficas) (Peña Marroquín, 2018, p. 20).

La técnica de recolección y análisis de los datos fue documental, para la cual se utilizó como instrumento de recolección de datos a publicaciones de Google donde se interpretó el programa de estudios Contaduría Pública de la Facultad de Ciencias Económicas y Empresariales de la Universidad Nacional de Canindeyú y el manual de los pronunciamientos internacionales, específicamente de los contenidos de los programas profesionales de formación en contaduría emitidas por la Federación Internacional de Contadores (International Federation of Accountants /IFAC) a través del Consejo de Normas Internacionales de Formación en Contaduría (International Accounting Education Standards Board/IAESB.

\section{RESULTADOS Y DISCUSIÓN}

Tabla 1. Componente en contaduría, finanzas y conocimientos relacionados 


\begin{tabular}{|c|c|}
\hline \multicolumn{2}{|c|}{ Contenidos de los programas profesionales de formación en Contaduría. } \\
\hline IES 2 & $\begin{array}{c}\text { Contaduría Pública FACEM- } \\
\text { UNICAN }\end{array}$ \\
\hline $\begin{array}{l}\text { (a) contaduría financiera y presentación de } \\
\text { informes; } \\
\text { (b) contaduría gerencial y control de gestión; } \\
\text { (c) tributación; } \\
\text { (d) derecho mercantil y societario; } \\
\text { (e) auditoría y aseguramiento; } \\
\text { (f) finanzas } \\
\text { y administración financiera; } \\
\text { (g) valores y ética profesionales. }\end{array}$ & $\begin{array}{l}\text { Área Profesional } \\
\text { a. Gabinete, } \\
\text { b. Contabilidad financiera I, II, III } \\
\text { c. Contabilidad de gestión I, II } \\
\text { d. Finanzas de empresas } \\
\text { e. Auditoria I, II } \\
\text { f. Auditoría gubernamental I, II } \\
\text { g. Contabilidad Gubernamental } \\
\text { h. Régimen legal de las empresas } \\
\text { i. Tributación I, II } \\
\text { j. Emprendedorismo } \\
\text { k. Ejercicio profesional } \\
\text { 1. Historia de la contabilidad } \\
\text { m. Calculo Mercantil } \\
\text { n. Contabilidad Bancaria } \\
\text { o. Contabilidad Agropecuaria } \\
\text { p. Convocatoria de Acreedores } \\
\text { q. Análisis de Estados Contables } \\
\text { r. Ética }\end{array}$ \\
\hline
\end{tabular}

Fuente. (Facultad de Ciencias Económicas y Empresariales de la Universidad Nacional de Canindeyú, 2020; Federación Internacional de Contadores \& Consejo de Normas Internacionales de Formación en Contaduría, 2008).

En la tabla 1 se observa que el plan de estudios de la carrera de Contaduría Pública cumple plenamente la recomendación de la IFAC en cuanto al contenido de programas profesionales de formación en contaduría.

En el plan de estudios de la carrera de define al conocimiento técnico profesional como aquellas disciplinas propias de la profesión y otras que complementan la formación y/o posibilitan un determinado énfasis en el área técnico-profesional de conformidad con el perfil de egreso. Comprenden las ciencias aplicadas que son relevantes para la carrera que permiten a los futuros profesionales apropiarse de los conocimientos y desarrollar las 
competencias necesarias para actuar en distintas instancias y modalidades de la profesión contable (Facultad de Ciencias Económicas y Empresariales de la Universidad Nacional de Canindeyú, 2020).

Tabla 2. Componente de conocimiento organizacional y de negocios

\begin{tabular}{|c|c|}
\hline \multicolumn{2}{|c|}{ Contenidos de los programas profesionales de formación en Contaduría. } \\
\hline IES 2 & Contaduría Pública FACEM-UNICAN \\
\hline $\begin{array}{l}\text { (a) economía; } \\
\text { (b) entorno de los negocios; } \\
\text { (c) gobernanza corporativa; } \\
\text { (d) ética de la empresa; } \\
\text { (e) mercados financieros; } \\
\text { (f) métodos cuantitativos; } \\
\text { (g) comportamiento organizacional; } \\
\text { (h) administración y la toma de decisiones } \\
\text { estratégicas; } \\
\text { (i) mercadeo y } \\
\text { (j) negocios internacionales y la } \\
\text { globalización. }\end{array}$ & $\begin{array}{l}\text { a. Microeconomía } \\
\text { b. Macroeconomía } \\
\text { c. Metodología y técnica de } \\
\text { investigación } \\
\text { d. Teoría de la administración } \\
\text { e. Relaciones Humanas. } \\
\text { f. Metodología del trabajo intelectual } \\
\text { g. Derecho del trabajo } \\
\text { h. Organización, Sistemas y Métodos } \\
\text { i. Seminario de Investigación } \\
\text { j. Administración Presupuestaria } \\
\text { k. Organización y Administración de } \\
\text { 1. Estadística } \\
\text { m. Comercio Exterior } \\
\text { n. Matemática para Contadores } \\
\text { o. Comunicación Oral y Escrita Español } \\
\text { p. Trabajo Final de Grado } \\
\text { /Guaraní }\end{array}$ \\
\hline
\end{tabular}

Fuente. (Facultad de Ciencias Económicas y Empresariales de la Universidad Nacional de Canindeyú, 2020; Federación Internacional de Contadores \& Consejo de Normas Internacionales de Formación en Contaduría, 2008) 
En cuanto al componente de conocimiento organizacional, se observa que la carrera cumple plenamente acorde a la IES, además, existen más materias que complementan el programa de estudios acorde a la recomendación de estudiantes, profesores y empleadores.

Tabla 3. Componente de la tecnología de la información

\begin{tabular}{|c|c|}
\hline \multicolumn{2}{|c|}{ Contenidos de los programas profesionales de formación en Contaduría } \\
\hline IES 2 & $\begin{array}{c}\text { Contaduría Pública FACEM- } \\
\text { UNICAN }\end{array}$ \\
\hline $\begin{array}{l}\text { (a) conocimiento general de la tecnología de la } \\
\text { información; } \\
\text { (b) conocimiento del control de la tecnología de } \\
\text { la información; } \\
\text { (c) competencias del control de la tecnología de } \\
\text { la información; } \\
\text { (d) competencias del usuario de la tecnología de } \\
\text { la información; y } \\
\text { (e) una o una mezcla de las competencias } \\
\text { correspondientes a las funciones gerenciales, de } \\
\text { evaluación y de diseño de los sistemas de } \\
\text { información. }\end{array}$ & $\begin{array}{ll}\text { a. Tecnología de } \\
\text { Información } \\
\text { Comunicación } \\
\text { b. Diseño de sistema de } \\
\text { información } \\
\text { c. Auditoria en Informática } \\
\text { d. Informática Aplicada }\end{array}$ \\
\hline
\end{tabular}

Fuente. (Facultad de Ciencias Económicas y Empresariales de la Universidad Nacional de Canindeyú, 2020; Federación Internacional de Contadores \& Consejo de Normas Internacionales de Formación en Contaduría, 2008)

En relación al componente de la tecnología de la información se observa que cumple con las instrucciones básicas de usuarios de tecnología, de hecho que a fondo le será difícil a un contador desarrollar un software para usuarios contables, pero en la carrera se cuenta con instrucciones básicas sobre los sistemas contables, es más se cuenta con un convenio con una empresa donde los estudiantes con los profesores simulan la contabilización de una empresa utilizando el sistema contable en la web sin necesidad de descargar en las maquinas. Esto ellos lo hacen dentro de las materias técnicos profesionales.

Por lo anterior, es importante resaltar que la carrera de Contaduría Pública está acreditada por la Agencia Nacional de Evaluación y Acreditación de la Educación Superior, el mismo es el órgano estatal responsable de evaluar y acreditar la calidad de las 
instituciones de educación superior en Paraguay, de las carreras de grado y programas de postgrado. La creación de la Agencia en el año 2003, en virtud de la Ley $\mathrm{N}^{\circ} 2072$, significó un importante adelanto del país hacia el aseguramiento de la calidad de la educación en este nivel educativo (Agencia Nacional de Evaluación y Acreditación de la Educación Superior, 2014, p. 7).

Menciona Seltzer, (2001). En la enseñanza de las Ciencias Contables adoptamos un perfil del campo de conocimiento menos técnico y más orientado hacia el gerenciamiento y la administración. La contabilidad puede servir a todas las personas, desde las economías domésticas a las economías más sofisticadas, incluso a nivel público y gubernamental. Con ella trabajan los empleados de los Bancos, de las oficinas, de empresas, los que administran organizaciones (escuelas, clubes, consorcios, hospitales, organismos públicos y políticos, etc). La contabilidad se planifica, se organiza, se administra, se regula.

Es por ello importante que los programas de estudios estén actualizados como resultado del trabajo en conjunto por parte de los empleadores, egresados, estudiantes, profesores y de toda la comunidad en sí.

Por otra parte, la realización de actividades docentes basadas en las técnicas y estrategias de investigación provocan que el alumno recree el conocimiento, favoreciendo la capacidad de resolución de problemas y casos prácticos, la comprensión de textos, la redacción de informes, tanto para los temas de estudio propuestos como para enfrentar el reto de seguir aprendiendo en su vida profesional de forma constante (Perez \& Perry, 2016).

Por consiguiente, la relación planteada entre sociedad y educación contable esboza un escenario con dos orientaciones: lo anhelado y lo impuesto. Según ese esquema, los estudiantes anhelan una educación contable que capte las necesidades del país y sus problemáticas, para avanzar con una mirada integral de las organizaciones y del saber contable (Ramírez Henao, 2017, p. 11).

Además, la formación de un profesional contador requiere conocimientos técnicos acorde a las necesidades laborales y en conformidad con los cambios legales y tributarios de cada nación (López Jara \& Cañizares Roig, 2018).

El Contador Público, como profesional en Ciencias Económicas, tiene el gran de desafío de perfeccionarse y actualizarse en forma permanente para lograr resultados eficientes, 
congruentes a las necesidades de las personas y entes que requieren de su asesoramiento o servicio. Ello implica necesariamente que el contador deberá, entre otras, formar su propio criterio profesional a la hora de tomar una decisión que involucre a terceros (Maida \& Perez Mercado, 2011).

La contabilidad ha sido básicamente la expresión de una relación social cuya esencia radica en una racionalidad controladora de la riqueza social que se produce y adscribe a una propiedad (Barrios Álvarez et al., 2010). Por tanto, es necesario realizar una revisión del componente de contabilidad que integra el currículo de los programas de contabilidad, enseñados por las universidades en todo el país, teniendo en consideración que sean reformulados y correspondan a los nuevos desafíos que enfrenta el contador en el desarrollo de la profesión (González, 2010, p. 7).

Los sistemas de información para la contabilidad y las tecnologías de la información y la comunicación (TIC) han estado siempre estrechamente vinculados con la Contabilidad (González Vidal \& Mas López, 2021, p. 1). Otras de las recomendaciones de la IES, es el conocimiento de las TIC de los contadores, en la Facultad de Ciencias Económicas y Empresariales se tiene un convenio con el sistema Starsoft en el que los estudiantes realizan sus prácticas contables a través de la materia Ejercicio Profesional donde se simulan el ejercicio de la profesión desde la carga de facturas hasta la emisión de los Estados Financieros (Facultad de Ciencias Económicas y Empresariales de la Universidad Nacional de Canindeyú, 2021).

Por consiguiente, Paraguay incorporó el sistema integrado de facturación electrónica nacional y cuál es la apreciación en su uso de los contribuyentes adheridos al plan piloto (Alvarez, 2021). Además, el país está en etapa de implementación de la facturación electrónica, el contador debe estar más preparado en tecnología y en la exigencias tributarias (Subsecretaría de Estado de Tributación, 2021).

Por ende, las enseñanzas de la carrera Contaduría Pública deben estar en consonancia a los cambios actuales, para eso se debe actualizar constantemente los contenidos programáticos de las carrera (Ron, 2002).

La contabilidad ha evolucionado extraordinariamente de la mano de la internacionalización y la globalización, hoy en día la contabilidad navega en otras aguas completamente distintas a la de hace algunos años, en los cuales el profesional contable se limitaba a presentar al usuario, información de tipo financiera y tributaria, sin generar 
valor agregado a las empresas, reteniéndolas en un mero conocimiento de su situación financiera sin transportarla al manejo profesional y eficiente de dicha información; es así, que la profesión contable de hoy, ha actualizado su metodología orientándose hacia esquemas de consultoría y asesoría gerencial, que ha permitido brindar a los usuarios de la información financiera soluciones completas y efectivas (Rodríguez, 2012).

El Consejo de Normas Internacionales de Formación en Contaduría facilita los contenidos mínimos que deben contener los programas de estudios, pero la Universidad debe adaptar estos contenidos y buscar las estrategias pedagógicas adecuadas para el desarrollo de los mismos buscando siempre de acuerdo a la realidad económico y social.

\section{CONCLUSIÓN}

Con base en los resultados encontrados se comprueba que la carrera de Contaduría Pública cumple plenamente las recomendaciones del manual de los pronunciamientos internacionales, específicamente de los contenidos de los programas profesionales de formación en contaduría.

Por lo anterior, la carrera tiene como propósito formar profesionales capaces de investigar, diseñar, analizar, asesorar, desarrollar y evaluar la información para la toma de decisiones con ética y responsabilidad social. Proporcionar a la sociedad contadores íntegros capaces de promover la investigación y promover innovación en cualquier ámbito de la sociedad. Por consiguiente, el egresado de la carrera de la Contaduría Pública debe ser un profesional que posee sólidos conocimientos científicos y técnicos, además debe poseer una formación integral que permiten elaborar información financiera de empresas, entidades públicas y privadas.

Se recomienda a la comunidad académica (estudiantes, docentes, directivos y empleadores) seguir con la excelencia y con la acreditación en cuanto a la certificación de la calidad de la Educación Superior que ayuda a cumplir con las normas nacionales de calidad y se comprueba que también se cumplen las normas internacionales, siempre actualizando los programas de estudios acorde a las demandas laborales y cambios de los organismos internacionales.

\section{REFERENCIAS BIBLIOGRÁFICAS}


Agencia Nacional de Evaluación y Acreditación de la Educación Superior. (2014). Modelo Nacional de Evaluación y Acreditación de la Educación Superior. http://www.aneaes.gov.py/aneaes/datos/Mecanismo/01_Mecanismo_de_evaluac ion_institucional.pdf

Alvarez, C. G. de. (2021). Implementación del sistema integrado de facturación electrónica nacional (SIFEN) en los contribuyentes en paraguay. Ciencias $\begin{array}{llll}\text { Económicas } & I S S N- & 2788-6425, & 2(4),\end{array}$ https://revistascientificas.una.py/index.php/reco/article/view/2206

Barrios Álvarez, C., Fúquene Sánchez, T., \& Lemos de la Cruz, J. E. (2010). Desarrollo de la investigación contable en el Centro Colombiano de Investigación Contable. Contaduría $\quad y \quad$ administración, 231, 151-177. http://www.scielo.org.mx/scielo.php?script=sci_abstract\&pid=S0186$\underline{10422010000200008 \& \operatorname{lng}=e s \& n r m=i s o \& t \operatorname{lng}=e s}$

Facultad de Ciencias Económicas y Empresariales de la Universidad Nacional de Canindeyú. (2011). Contaduría Pública-Plan 2020. https://www.facem.edu.py/index.php/academicas/carreras/contabilidad/contadur \%C3\%ADa-p\%C3\%BAblica-plan-2020

Facultad de Ciencias Económicas y Empresariales de la Universidad Nacional de Canindeyú. (2020). Proyecto de Adecuación y Mejoras del Plan de Estudio de la Carrera de Contaduría Pública (Universidad Nacional de Canindeyú). https://www.facem.edu.py/index.php/academicas/carreras/contabilidad/contadur \%C3\%ADa-p\%C3\%BAblica-plan-2020

Facultad de Ciencias Económicas y Empresariales de la Universidad Nacional de Canindeyú. (2021). Capacitación a Docentes sobre sistema Contable. https://www.facem.edu.py/index.php/11-noticias/409-capacitaci\%C3\%B3n-adocentes-sobre-sistema-contable

Federación Internacional de Contadores, \& Consejo de Normas Internacionales de Formación en Contaduría. (2008). Manual de los pronunciamientos internacionales de formación. https://www.ifac.org/system/files/downloads/Spanish_Translation_Normas_Inte rnacionales_de_Formacion_2008.pdf 
García Jiménez, M. D. (2014). Enseñanza de la contabilidad como disciplina académica: Concepciones de ciencias del profesorado y pensamiento crítico. Entramado, $10(1)$, 164-174.

http://www.scielo.org.co/scielo.php?script=sci_abstract\&pid=S1900$\underline{38032014000100010 \& \operatorname{lng}=\mathrm{en} \& n \mathrm{~nm}=\mathrm{iso} \& \mathrm{t} \operatorname{lng}=\mathrm{es}}$

González, P. G. (2010). Avances y desafíos sobre la enseñanza de la contabilidad de gestión en Colombia: desde una perspectiva de mi pymes y de la educación superior. Revista Universo Contábil, 140-155. https://doi.org/10.4270/ruc.2010327

González Vidal, I. M., \& Mas López, C. J. (2021). Nuevo enfoque en ciencia, matemáticas, ingeniería y tecnología en la enseñanza de los sistemas de información para la contabilidad. Cofin Habana, 15(2). http://scielo.sld.cu/scielo.php?script=sci_abstract\&pid=S2073$\underline{60612021000200007 \& \operatorname{lng}=e s \& n r m=i s o \& t \operatorname{lng}=e s}$

Hernandez Sampieri, R., Fernandez Collado, C., \& Pilar Baptista, L. (2014). Metodología de la Investigación (6 ed.). McGRAW-HILL.

Hernandez Sampieri, R., \& Mendoza Torres, C. P. (2018). Metodología de la investigación. Mc Graw Hill. 978-1-4562-6096-5

López Jara, A. A., \& Cañizares Roig, M. (2018). Diagnóstico sobre la metodología para la enseñanza de la Contabilidad en la Universidad Católica de Cuenca, Sede Macas. 13. http://scielo.sld.cu/pdf/rces/v38n1/0257-4314-rces-38-01-e5.pdf

Maida, A. K., \& Perez Mercado, G. C. (2011). La formación del criterio profesional del contador y su importancia en el campo laboral. 107. https://bdigital.uncu.edu.ar/objetos_digitales/5296/maidalaformacindelcriterioprofesional.pdf

Müggenburg, M. C., \& Pérez, I. (2018). Tipos de estudio en el enfoque de investigación cuantitativa. Enfermería Universitaria, 4(1). https://www.redalyc.org/pdf/3587/358741821004.pdf

Peña Marroquín, R. (2018). METODOLOGÍA DE LA INVESTIGACIÓN. 26. http://www.une.edu.pe/Sesion04-Metodologia_de_la_investigacion.pdf 
Perez, V. G., \& Perry, G. R. P. (2016). La investigación contable como herramienta didáctica en la docencia de la contabilidad. Ciencias Administrativas, 7, 37-47. https://www.redalyc.org/journal/5116/511652569006/html/

Ramírez Henao, D. F. (2017). Análisis y desarrollo de la educación contable: Temáticas de su abordaje y principales problemáticas según el pensamiento estudiantil socializado por la Fenecop (2000-2016). http://www.scielo.org.co/pdf/cuco/v18n46/0123-1472-cuco-18-46-00014.pdf

Rodríguez, J. M. (2012). La contabilidad en el contex to de la globalización y la revolución teleinformática. 24. https://www.redalyc.org/pdf/364/36424414008.pdf

Ron, G. (2002). Algunas estrategias de enseñanzas-aprendizaje aplicadas a la contabilidad. Escritos $\quad$ Contables, $43, \quad$ 59-73. http://bibliotecadigital.uns.edu.ar/scielo.php?script=sci_abstract\&pid=S151442752002001100003\&lng=es\&nrm=iso\&tlng=es

Salvioni Durañona, T. M. (2020). Contaduría pública: Semejanzas y diferencias entre las mallas curriculares de la Universidad Nacional de Asunción y de la Universidad Nacional Autónoma de México. Población y Desarrollo, 26(50), 96-105. http://scielo.iics.una.py/scielo.php?script=sci_arttext\&pid=S2076$\underline{054 X 2020005000096 \& \operatorname{lng}=\mathrm{es} \& \mathrm{nrm}=\mathrm{iso} \& \operatorname{tn} \mathrm{ln}=\mathrm{es}}$

Seltzer, J. $\quad$ C. (2001). Contabilidad y docencia. https://rieoei.org/historico/deloslectores/711Seltzer.PDF

Subsecretaría de Estado de Tributación. (2021). Resolucion General No 105/2021 «Por la cual se disponen medidas administrativas para la emisión de documentos tributarios electrónicos y se designan a contribuyentes como facturadores electrónicos»._https://www.set.gov.py/ 